\title{
Ultrastructural Analyses of Deciduous Teeth Affected by Hypocalcified Amelogenesis Imperfecta from a Family with a Novel Y458X FAM83H Nonsense Mutation
}

\author{
W. El-Sayed ${ }^{a, b}$ R.C. Shore ${ }^{a} \quad$ D.A. Parry ${ }^{b} \quad$ C.F. Inglehearn ${ }^{b} \quad$ A.J. Mighell ${ }^{a}$ b \\ a Leeds Dental Institute, and ${ }^{b}$ Leeds Institute of Molecular Medicine, University of Leeds, Leeds, UK
}

\section{Key Words}

Amelogenesis imperfecta · FAM83H - Enamel • Deciduous teeth $\cdot$ Scanning electron microscopy

\begin{abstract}
Background: Nonsense mutations in FAM83H are a recently described underlying cause of autosomal dominant (AD) hypocalcified amelogenesis imperfecta (Al). Objective: This study aims to report a novel c.1374C >A p.Y458X nonsense mutation and describe the associated ultrastructural phenotype of deciduous teeth. Methods: A family of European origin from the Iberian Peninsula with AD-inherited Al was ascertained. Family members were assessed through clinical examination and supporting investigations. Naturally exfoliated deciduous teeth from 2 siblings were investigated by scanning electron microscopy (SEM), energy dispersive X-ray analysis (EDX) and transverse microradiography (TMR). $\boldsymbol{R} \boldsymbol{e}$ sults: On clinical and radiographic investigation the appearances of the affected deciduous and permanent teeth were consistent with hypocalcified Al with small focal areas of more normal looking enamel. DNA sequencing identified a novel c.1374C >A p.Y458X FAM83H nonsense mutation in affected, but not in either unaffected family members or unrelated controls. Exfoliated teeth were characterised by substantial post-eruptive enamel loss on gross examination. Irregular, poor quality enamel prisms were observed on SEM. These were coated in amorphous material. TMR and EDX
\end{abstract}

confirmed reduced mineral and increased organic content in enamel, respectively. Conclusions: FAM83H nonsense mutations have recently been recognised as a cause of $A D$ hypocalcified AI. We report a novel nonsense $F A M 83 \mathrm{H}$ mutation and describe the associated preliminary ultrastructural phenotype in deciduous teeth. This is characterised by poorly formed enamel rods with inappropriate retention of amorphous material, which is likely to represent retained organic matrix that contributes to the overall hypomineralised phenotype.

Copyright $\odot 2009$ S. Karger AG, Basel

\section{Introduction}

The tooth crown is covered by non-vital, acellular enamel that is unique among biomineralised tissues because of its high mineral content, large crystals, and or-

\section{Abbreviations used in this paper}

AD autosomal dominant

AI amelogenesis imperfecta

DEJ dentino enamel junction

EDX energy dispersive X-ray analysis

SEM scanning electron microscopy

TMR transverse microradiography

\section{KARGER}

Fax +4161306 1234

E-Mail karger@karger.ch

www.karger.com (c) 2009 S. Karger AG, Basel

$1422-6405 / 10 / 1913-0235 \$ 26.00 / 0$

Accessible online at:

www.karger.com/cto
Dr. Alan J. Mighell

Department of Oral Medicine

Leeds Dental Institute, University of Leeds

Clarendon Way, Leeds LS2 9LU (UK)

Tel. +44 113343 6121, Fax +44 113343 6165, E-Mail a.j.mighell@leeds.ac.uk 
ganised prism structure. Enamel development (amelogenesis) progresses through three stages: secretory, transition, and maturation [Warshawsky and Smith, 1974; Nanci and Smith, 1992; Smith et al., 1995]. During the secretory stage, the columnar-shaped ameloblasts secrete a protein-rich, partially mineralised, self-organising enamel matrix. There is rapid formation of thin, needle-like hydroxyapatite crystals that account for $30 \%$ by volume of the enamel matrix and elongate into long thin ribbons [Kirkham et al., 1988; Cuisinier et al., 1992]. When the matrix reaches full thickness, the secretory stage ends and the ameloblasts enter the transition stage and reduce in size. Matrix is then removed by proteases with associated individual crystal growth during the maturation stage until the enamel attains its final hardened state [Lu et al., 2008]. These general features of amelogenesis are remarkably consistent among different species [Robinson et al., 1988].

Amelogenesis imperfecta (AI) is a genetically and clinically heterogeneous group of inherited conditions typically characterised by generalised enamel defects of both primary and permanent dentitions [Witkop, 1988; Aldred et al., 2003]. Mutations in the genes encoding enamel matrix proteins AMELX (MIM 301200) and ENAM (MIM 608563), and enamel matrix modifying proteases MMP20 (MIM 612529) and KLK4 (MIM 204700) cause AI. Recently eight nonsense mutations causing AI have been reported in FA M83H (MIM 130900), a gene of unknown function: c.891T >A, p.Y297X; c.973C/ T, p.R325X; c.1192C/T, p.Q398X; c.1243G>T, p.E415X; c.1330C.T, p.Q444X; c.1366C.T, p.Q456X; c.1380G>A, p.W460X; and c.2029C>T, p.Q677X [Kim et al., 2008; Lee et al., 2008; Hart et al., 2009]. These mutations cause hypocalcified AI, a form of AI in which enamel biomineralisation is incomplete. In this study we report a novel nonsense FAM83H mutation (c.1374C>A; p.Y458X) and described the associated preliminary ultrastructural phenotype in affected deciduous teeth.

\section{Subjects and Methods}

A family of European origin from the Iberian Peninsula with autosomal dominant (AD)-inherited hypocalcified AI was ascertained during delivery of dental care at Leeds Dental Institute. Peripheral blood samples were obtained from affected and unaffected participating family members and genomic DNA prepared by conventional salting methods. A panel of 96 control DNAs of European descent were used. All samples were obtained with informed consent under ethical approval from the Leeds (West) NHS Trust Ethics committee.
FAM83H Mutation Analysis

All 5 exons and exon/intron boundaries of the FAM83H gene were PCR-amplified using oligonucleotide primers described by Kim et al. [2008]. Purified PCR products were sequenced using the Big Dye terminator Kit v.3.1 (Applied Biosystems, Forster City, Calif., USA) and separated by electrophoresis on an ABI 3130 XL DNA analyser. The sequence produced was analysed using the ABI Prism sequence Analyser and SeqScape software packages (Applied Biosystems).

\section{Scanning Electron Microscopy, Energy Dispersive X-Ray}

Spectroscopy and Transverse Microradiography

Standard methods were used for tooth section $(100 \mu \mathrm{m})$ preparation from exfoliated deciduous teeth of 2 affected individuals and normal control teeth, which were then investigated by scanning electron microscopy (SEM), energy dispersive X-ray spectroscopy (EDX) and transverse microradiography (TMR) [Shore et al., 2002; Barron et al., 2008]. Microstructural analysis was undertaken using a Jeol 35 SEM fitted with the Deben Genie upgrade (Deben Engineering, Debenham, UK). EDX elemental analysis was performed using a detector fitted with an ultrathin window and driven by WinEDX 3 software (Thomson Scientific, Carlton, Australia). TMR involved sampling across sections from 2 affected and 2 control teeth at a minimum of 10 different points each within the enamel.

\section{Results}

\section{Clinical Phenotype and FAM83H Mutation}

A typical hypocalcified AI clinical phenotype was observed in the proband of a family with 14 reported affected individuals and an AD pattern of inheritance (fig. 1). The enamel was pigmented, exhibiting yellowish to brownish discoloration that progressed from a paler, cream colour on initial tooth eruption. There was widespread evidence of post-eruptive enamel loss due to attrition and enamel fractures. This resulted in enamel with an irregular rough surface and the remaining enamel was softer than normal. The cervical enamel was most typically spared, but was also prone to loss and failure. Focal islands of apparently more normal enamel were present and were particularly evident on the cusps of some posterior teeth (fig. 1A).

Dental radiographs were characterised by a reduced distinction in radiodensity between the enamel and dentine, compared to that observed in normal teeth where enamel is obviously more radiodense (fig. 1B). The teeth were sensitive to thermal stimuli. A nonsense mutation (c.1374C >A, p.Y458X) wasidentified in exon 5 of FAM83H (forward and reverse strands) in affected, but not the unaffected family member tested or normal control patients (fig. 1C). 

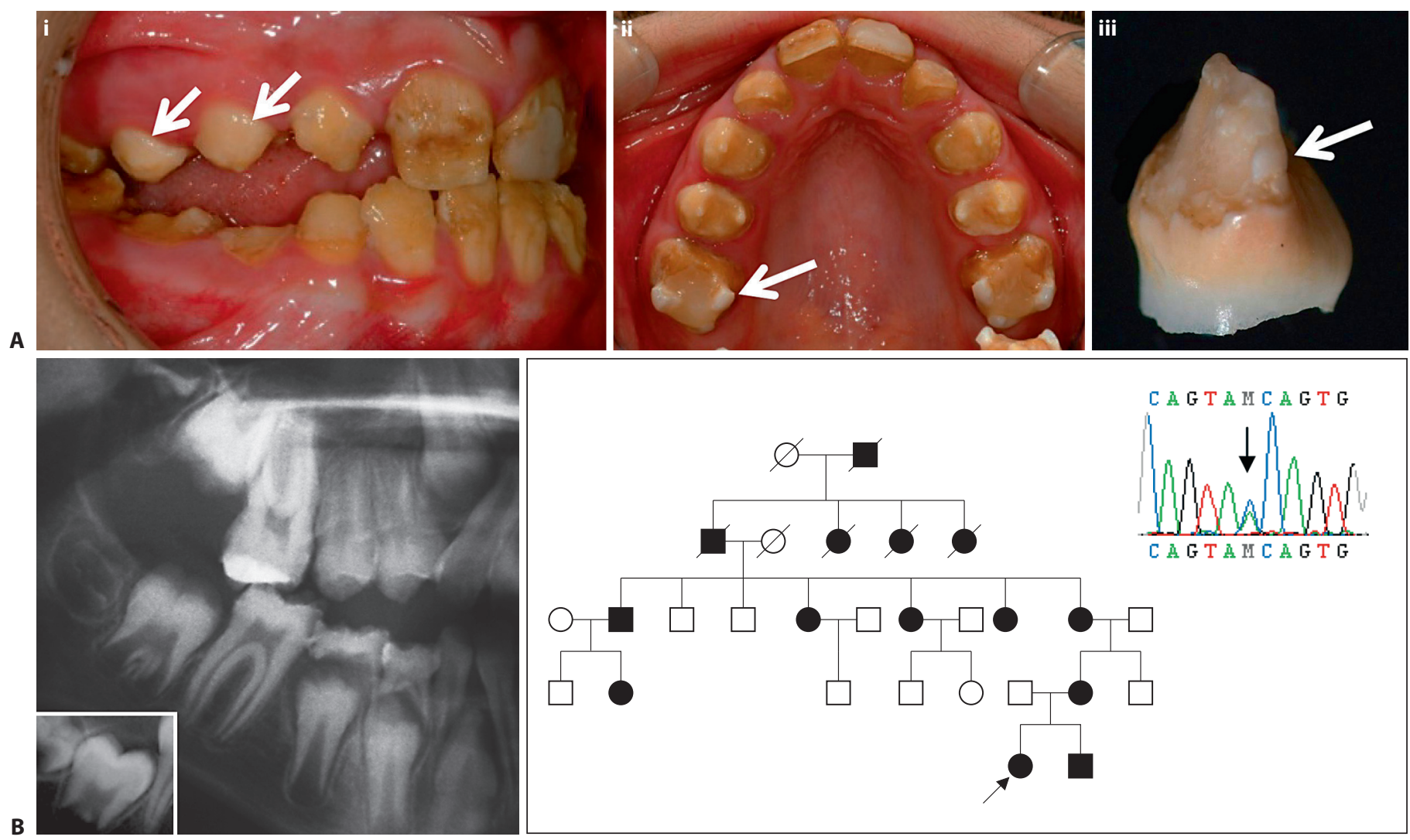

Fig. 1. Clinical and radiographic phenotype, pedigree and FAM83H mutation. A Mixed dentition of the 10 -year-old proband. (i) The buccal surfaces of the erupting permanent premolar teeth (arrows) are paler and exhibit less post-eruptive enamel loss compared to teeth that have been present in the mouth for longer. There is a malocclusion that in part reflects the transition from deciduous to permanent dentition. (ii) Focal islands of pale, harder enamel are most obvious at the cusp tips of the first permanent molar teeth (arrow) and to a lesser extent involve the cusp tips of the permanent premolar teeth. The permanent upper left central incisor labial face has been restored. (iii) An exfoliated deciduous incisor tooth exhibits typical loss of most of the enamel with relative sparing of the cervical enamel. A small island of harder enamel has persisted close to the DEJ (arrow). B Detail from a panoramic radiograph of the proband. Unerupted teeth have a normal morphology, but the enamel is characterised by diminished radiodensity that contrasts from that observed in normal teeth (inset box). Erupted teeth exhibit significant premature post-eruptive loss of enamel. C Family pedigree and confirmation of the c.1374C $>$ A mutation.

\section{Ultrastructural Analyses of Deciduous Teeth}

Little enamel remained on exfoliated deciduous teeth, with most of the retained enamel localised to the cervical areas (fig. 1A). SEM examination of sections of affected teeth revealed loss of normal enamel architecture, which in part reflected the presence of an amorphous material obscuring the enamel rods (fig. 2A). It was typically distributed over most of the enamel, but was particularly evident at the dentino enamel junction (DEJ). The enamel rods were incompletely formed. The underlying dentine observed by SEM had normal architecture. TMR sampling identified a mean mineral percentage in affected enamel of $50.8 \pm 2.9 \%$ compared to the mean for control teeth of $87 \pm 2.2 \%$. The difference between these two values is statistically significant ( $\mathrm{p}<$ $0.001, \mathrm{t}$ test).

The elemental analysis by EDX identified an increased carbon:oxygen ratio where the amorphous material was present (fig. 2B). By contrast in areas where enamel rods were more easily observed, the carbon:oxygen ratio was reduced, but not to levels seen in control teeth. A small but consistent increase in the nitrogen peak was evident throughout affected areas, compared to that observed in control teeth. The calcium:phosphorous ratios were similar in affected and control teeth. 
A
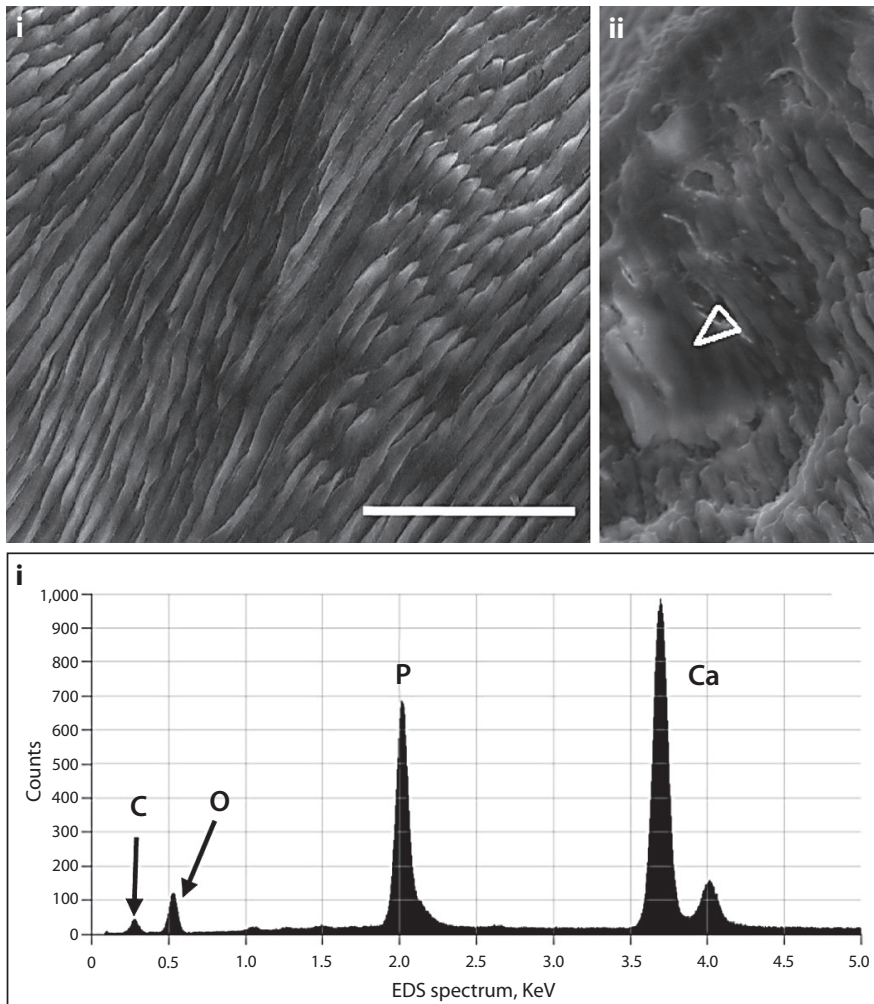

B

Fig. 2. Ultrastructural phenotyping of deciduous teeth. A SEM of exfoliated deciduous tooth cervical enamel. The appearances of control enamel (i) contrast with the poorly formed enamel rods that are partially obscured by amorphous material (open arrow heads) in affected incisor (ii) and molar (iii) teeth. $\mathrm{R}$ is embedding

\section{Discussion}

FAM83H mutations were first described as a cause of AD-hypocalcified AI in 2008, with subsequent description of eight different mutations in families of diverse ethnic origins [Kim et al., 2008; Lee et al., 2008; Hart et al., 2009]. The clinical tooth phenotype observed here was consistent with those previously reported. In particular, the observation of small islands of more normal looking and persistent enamel appears to be a distinctive clinical marker of exon 5 FAM83H nonsense mutations. The foci of harder enamel occur at random sites and do not follow an obvious pattern. Localisation of the enamel foci to cusp tips has not been previously reported and this is not confirmed as a reliable clinical marker of FAM83H mutations. Normal radiographic crown morphology of unerupted teeth supports formation of a near-normal volume of enamel matrix. It remains unknown whether the enamel matrix produced by the ameloblasts has a nor- resin and not dental tissue; bar $50 \mu \mathrm{m}$. B EDX spectra. In control enamel (i), the C:O ratio is low compared to that in affected enamel (ii). A small $\mathrm{N}$ peak was observed (between the $\mathrm{C}$ and $\mathrm{O}$ peaks) in affected, but not control teeth. Similar peaks are observed in affected and control teeth for $\mathrm{Ca}$ and $\mathrm{P}$. mal composition or not at the different stages of amelogenesis. Failure of normal enamel matrix processing is recognised to cause abnormal enamel and a hypomineralised phenotype [Hart et al., 2004; Kim et al., 2005], but may not be relevant in this instance. Accordingly, it cannot be stated with certainty at which stage of amelogenesis FAM83H mutations exert their detrimental effect.

The novel FAM83H mutation identified in this study causes a premature stop codon in exon 5 , in common with those previously reported. This is consistent with the hypothesis that the FAM83H protein carboxy-terminus plays an important role in amelogenesis. The mutation described herein would result in a truncated protein that is 721 amino acids shorter than the wild-type.

The reported mutations are broadly spread across exon 5 , although the mutation identified in this study is close to two others and may indicate a mutation hotspot within a gene that is prone to inheritable mutations in exon 5 . The mechanisms by which exon 5 FAM $83 \mathrm{H}$ nonsense muta- 
tions result in hypocalcified enamel, which includes focal islands of harder enamel, is currently unknown. It possibly reflects haploinsufficiency that in part may be due to mRNA nonsense mediated decay or a hypomorphic partial loss of function. Alternatively, the resultant mutant protein may have a dominant negative effect or lead to rapid degradation of all FAM83H and a null phenotype. Other, as yet unidentified genes may allow a partial, but largely ineffectual rescue of the enamel phenotype. FAM83H is widely expressed, but mutations are only recognised to cause abnormalities of enamel formation.

Early clinical failure of the enamel is not surprising given SEM observation of poorly and incompletely formed enamel rods with inappropriate retention of organic material supported by EDX. This provides new insight into the detrimental impact on enamel formation of a FAM83H mutation. Similar observations have been reported in teeth described clinically as hypomaturation AI in non-syndromic forms (of uncertain genotype) [Wright et al., 1992; Shore et al., 2002] and in Jalili syndrome caused by mutations in CNNM4, encoding a putative $\mathrm{Mg}^{2+}$ transporter [Parry et al., 2009].

FAM83H is expressed in ameloblasts where its functions remain obscure. Sequence homology analyses indi- cate that FAM83H possibly includes a phospholipase Dlike domain. Accordingly, FAM83H may be involved in signal transduction or lipid metabolism. Inappropriate retention of lipid or lipid/protein complexes within the enamel matrix has been proposed as a possible mechanism central to some forms of AI clinically diagnosed as the hypomaturation subtype [Shore et al., 2002]. In the teeth presented here, the marked increase in carbon in particular may be consistent with elevated lipid content, at least in the most markedly affected areas. The less marked but more generalised increase in nitrogen may indicate a more widespread retention of protein throughout the enamel. Elucidating the functions of FAM83H can be expected to provide novel and important insight into biomineralisation.

\section{Acknowledgements}

The authors thank the family for their participation and $\mathrm{Mr}$. Stephen Fayle, Department of Child Dental Health, Leeds Dental Institute, for information about the family. This work was funded by the Egyptian Government (WE) and The Wellcome Trust (grant numbers 082448 and 075945).

\section{References}

Aldred, M.J., R. Savarirayan, P.J. Crawford (2003) Amelogenesis imperfecta: a classification and catalogue for the 21st century. Oral Dis 9: 19-23.

Barron, M.J., S.J. Brookes, C.E. Draper, D. Garrod, J. Kirkham, R.C. Shore, M.J. Dixon (2008) The cell adhesion molecule nectin-1 is critical for normal enamel formation in mice. Hum Mol Genet 17: 3509-3520.

Cuisinier, F.J., P. Steuer, B. Senger, J.C. Voegel, R.M. Frank (1992) Human amelogenesis. I: High resolution electron microscopy study of ribbonlike crystals. Calcif Tissue Int 51: 259-268.

-Hart, P.S., S. Becerik, D. Cogulu, G. Emingil, D. Ozdemir-Ozenen, S.T. Han, P.P. Sulima, E. Firatli, T.C. Hart (2009) Novel FAM83H mutations in Turkish families with autosomal dominant hypocalcified amelogenesis imperfecta. Clin Genet 75: 401-404.

Hart, P.S., T.C. Hart, M.D. Michalec, O.H. Ryu, D. Simmons, S. Hong, J.T. Wright (2004) Mutation in kallikrein 4 causes autosomal recessive hypomaturation amelogenesis imperfecta. J Med Genet 41: 545-549.

Kim, J.W., S.K. Lee, Z.H. Lee, J.C. Park, K.E. Lee, M.H. Lee, J.T. Park, B.M. Seo, J.C. Hu, J.P. Simmer (2008) FAM83H mutations in families with autosomal-dominant hypocalcified amelogenesis imperfecta. Am J Hum Genet 82: $489-494$.
Kim, J.W., J.P. Simmer, T.C. Hart, P.S. Hart, M.D. Ramaswami, J.D. Bartlett, J.C. Hu (2005) MMP-20 mutation in autosomal recessive pigmented hypomaturation amelogenesis imperfecta. J Med Genet 42: 271-275.

Kirkham, J., C. Robinson, J.A. Weatherell, A. Richards, O. Fejerskov, K. Josephsen (1988) Maturation in developing permanent porcine enamel. J Dent Res 67: 1156-1160.

Lee, S.K., J.C. Hu, J.D. Bartlett, K.E. Lee, B.P. Lin, J.P. Simmer, J.W. Kim (2008) Mutational spectrum of FAM83H: the C-terminal portion is required for tooth enamel calcification. Hum Mutat 29: E95-E99.

Lu, Y., P. Papagerakis, Y. Yamakoshi, J.C. Hu, J.D. Bartlett, J.P. Simmer (2008) Functions of KLK4 and MMP-20 in dental enamel formation. Biol Chem 389: 695-700.

Nanci, A., C.E. Smith (1992) Development and calcification of enamel; in: Mineralization in Biological Systems. Boca Raton, CRC Press, pp 313-343.

Parry, D.A., A.J. Mighell, W. El-Sayed, R.C. Shore, I.K. Jalili, H. Dollfus, A. Bloch-Zupan, R. Carlos, I.M. Carr, L.M. Downey, K.M. Blain, D.C. Mansfield, M. Shahrabi, M. Heidari, P. Aref, M. Abbasi, M. Michaelides, A.T. Moore, J. Kirkham, C.F. Inglehearn (2009) Mutations in CNNM4 cause Jalili syndrome, consisting of autosomal- recessive cone-rod dystrophy and amelogenesis imperfecta. Am J Hum Genet 84: 266-273.

Robinson, C., J. Kirkham, J.A. Weatherell, A Richards, K. Josephsen, O. Fejerskov (1988) Mineral and protein concentrations in enamel of the developing permanent porcine dentition. Caries Res 22: 321-326.

-Shore, R.C., B. Backman, S.J. Brookes, J. Kirkham, S.R. Wood, C. Robinson (2002) Inheritance pattern and elemental composition of enamel affected by hypomaturation amelogenesis imperfecta. Connect Tissue Res 43: 466-471.

Smith, C.E., W.Y. Chen, M. Issid, A. Fazel (1995) Enamel matrix protein turnover during amelogenesis: basic biochemical properties of short-lived sulfated enamel proteins. Calc Tissue Int 57: 133-144.

-Warshawsky, H., C.E. Smith (1974) Morphological classification of rat incisor ameloblasts. Anat Rec 179: 423-446.

Witkop, C.J. Jr (1988) Amelogenesis imperfecta, dentinogenesis imperfecta and dentin dysplasia revisited: problems in classification. J Oral Pathol 17: 547-553.

Wright, J.T., V. Lord, C. Robinson, R. Shore (1992) Enamel ultrastructure in pigmented hypomaturation amelogenesis imperfecta. J Oral Pathol Med 21: 390-394. 